Pramāna, Vol. 15, No. 4, October 1980, pp. 327-340. (C) Printed in India.

\title{
Embedding of non-simple Lie groups, coupling constant relations and non-uniqueness of models of unification
}

\author{
J PASUPATHY and E C G SUDARSHAN* \\ Centre for Theoretical Studies, Indian Institute of Science, Bangalore 560 012, India \\ * Permanent address: Centre for Particle Theory and Department of Physics, \\ University of Texas, Austin, Texas 78712, USA
}

MS received 2 June 1980

\begin{abstract}
A general derivation of the coupling constant relations which result on embedding a non-simple group like $\mathrm{SU}_{L}(2) \otimes \mathrm{U}(1)$ in a larger simple group (or graded Lie group) is given. It is shown that such relations depend only on the requirement (i) that the multiplet of vector fields form an irreducible representation of the unifying algebra and (ii) the transformation properties of the fermions under $\mathrm{SU}_{L}(2)$. This point is illustrated in two ways, one by constructing two different unification groups containing the same fermions and therefore have same Weinberg angle; the other by putting different $\mathrm{SU}_{L}(2)$ structures on the same fermions and consequently have different Weinberg angles. In particular the value $\sin ^{2} \theta=3 / 8$ is characteristic of the sequential doublet models or models which invoke a large number of additional leptons like $E_{6}$, while addition of extra charged fermion singlets can reduce the value of $\sin ^{2} \theta$ to $1 / 4$. We point out that at the present time the models of grand unification are far from unique.
\end{abstract}

Keywords. Coupling constant relations; embedding in higher symmetries; Weinberg-Salam model; grand unified theories; Shmushkevich relations; Weinberg angle; neutral currents; Schur's lemma; graded Lie groups.

\section{Introduction}

One of the deficiencies of the standard gauge-model for electroweak interactions (Weinberg 1967; Salam 1968) is that the minimal theory based on the gauge group $S U_{L}(2) \otimes U(1),\left(G l a s h o w 1961\right.$ ) involves two coupling constants $g$ and $g^{\prime}$ whose relative magnitude is parametrised by the Weinberg angle

$$
\tan \theta_{w}=g / g^{\prime}
$$

remains an arbitrary parameter. Current experiments (Dydak 1979) give the value

$$
\sin ^{2} \theta_{w}=0.23 \pm 0.02
$$

The natural way to remove this arbitrariness is to embed this group in a higher symmetry group (i.e. Lie group or a graded Lie group cf. below) which involves only one coupling constant so that the couplings $g$ and $g^{\prime}$ corresponding to the $\mathrm{SU}_{L}(2) \otimes$ $\mathrm{U}$ (1) subgroup of this higher symmetry, become algebraically related.

Theoretical attempts at this embedding have in the past involved the hadron and lepton sectors separately, while more recently, the so-called grand unified theories (Pati and Salam 1973; Georgi and Glashow 1974) have received increasing attention. 
The chief virtue of these latter models is their prediction of the value of $\sin ^{2} \theta_{w}$. These models have other predictions like the proton decay rate, baryon excess in the universe, mass relations between quarks and leptons of different generations, etc. But these latter predictions cannot be considered critical tests of the model, due to paucity of experimental data as well as theoretical uncertainties. Besides, the question of the correct gauge group for grand unification is not settled for a variety of reasons.

(i) The question of the correct gauge group for the electroweak interactions itself is still open. For example the left-right symmetric model for the electroweak interactions (Bajaj and Rajasekaran 1979 and references cited therein) is in as good an agreement with the currently available experimental data as the minimal $\mathrm{SU}_{L}(2) \otimes U(1)$.

(ii) At present, there are insufficient experimental grounds to discriminate between the fractionally charged and integrally charged quark models for strong interactions.

(iii) No understanding of the generations puzzle for quarks and leptons exists at present. There are rival models which are sensitive to questions like whether the quark generation follow the orthodox doublet pattern $(u, d) ;(c, s) ;(t, b) ; \ldots$ or triplets like $(p, n, \Lambda) ;\left(p^{\prime}, n^{\prime}, \Lambda^{\prime}\right) ; \ldots$ required as for example by the $E_{6}$ model (cf. $\left.\S 3\right)$.

(iv) Even if one makes specific choices for the basic fermion multiplets, the electric charge and the $\mathrm{SU}_{L}(2)$ isospin assignments, there are several unifying algebras which can accommodate a given fermion multiplet.

Further, during the last year, theories have been introduced (Neeman 1979; Fairlie $1979)$ based on the graded Lie group $S U(2 / 1)$ which have the virtue (?) of not putting quarks and leptons in the same multiplet ${ }^{\dagger}$ but at the price of introducing gauge fields which are more complex than the usual ones.

From the above discussion it is clear that the search for a symmetry larger than $\mathrm{SU}_{L}(2) \otimes \mathrm{U}(1)$ is wide open. One is then led to ask (i) whether the unification result for the mixing angle depends critically on the specific choice of the larger symmetry group, or (ii) whether it depends on the colour symmetry for the quarks being broken or unbroken and the choice of the electroweak group itself.

In this paper we shall be concerned with algebraic relations between coupling strengths obtained by embedding a non-simple Lie group in a higher symmetry group. Although our results are valid more generally, we shall discuss gauge theories mainly, since these are most interesting.

In $\$ 2$ we derive some simple mathematical relations and show that the unification result for $\sin ^{2} \theta_{w}$ depends only on the set of fermions and their classification under the observed group. This of course applies to other coupling constant relations as well.

This point is illustrated in $\S 3$ in two different ways. We first show that the same set of fermions, given the same $\mathrm{SU}_{L}(2)$ transformation law can be embedded in different algebraic structures yielding the same value for the Weinberg angle. This is reinforced by considering different models which have the same set of fermions and grand unifying group but have different $\mathrm{SU}_{L}$ (2) transformation law for fermions and so have different values of $\theta_{w}$. A brief discussion of $\operatorname{SU}(5), \operatorname{SO}(10)$, and $E_{6}$ models is given. The question of broken versus unbroken colour symmetry is considered next in the context of 4 quarks and 4 leptons.

tAlthough the point is extraneous to our main theme, it is worth emphasizing, that grand unification in the sense of putting quarks and leptons in the same multiple is not forced on us by considerations of renormalizability as in the case of electroweak unification. 
Enlarging the $\mathrm{SU}_{L}(2) \otimes \mathrm{U}(1)$ group by the process of embedding, usually but not always leads to the introduction of extra neutral current generators. Brief comments are made in $\$ 4$ on the role of these in comparing the models with experiment are made.

An appendix provides a discussion of representation theory of graded Lie algebras.

In a recent paper Bajaj and Rajasekaran (1980a) have also derived some of our results on algebraic consequences of Grand unification. Our present work provides a more complete discussion, not only in the context of Lie Groups, but also in the context of more general algebraic structures. Further we establish the nontriviality of the observation that coupling constant relations obtained by unification, do not necessarily depend on the unification group, by explicit illustration with specific models $(\S 3)$.

\section{Smushkevich relations ${ }^{\dagger}$}

We begin by proving the following proposition: Let $\psi_{a}$ be a unitary irreducible representation of a group $G$ and let $\Gamma_{a b}^{a}$ be the coupling coefficients which are needed to make the interaction Lagrangian

$$
\mathscr{L}_{I}=\sum_{a b} g \bar{\psi}_{a} \Gamma_{a b}^{a} \psi_{b} V_{a},
$$

and invariant under $G . \quad V_{\alpha}$ is a set of vector fields forming an irreducible unitary representation of $G$. Here and in the following we suppress space-time indices as well as factors like, $\gamma_{\mu}\left(1 \pm \gamma_{5}\right)$. Then

$$
\begin{aligned}
& \sum_{a b}\left|\Gamma_{a b}^{a}\right|^{2} \text { is independent of } a, \\
& \sum_{a b} \Gamma_{a b}^{a} \Gamma_{a b}^{\beta^{*}} \text { vanishes for } \alpha \neq \beta,
\end{aligned}
$$

Proof: Let $\mathscr{D}_{a a^{\prime}}(g)$ be the representation matrix $(g \in G)$ with

$$
\begin{aligned}
& \psi_{a} \rightarrow \psi_{a}^{\prime}=\mathscr{D}_{a a^{\prime}}(g) \psi_{a^{\prime}}, \\
& \bar{\psi}_{a} \rightarrow \bar{\psi}_{a}^{\prime}=\mathscr{D}_{a a^{\prime}}^{*}(g) \bar{\psi}_{a^{\prime}}, \\
& V_{a} \rightarrow V_{a}^{\prime}=d_{a a^{\prime}}(g) V_{a^{\prime}} .
\end{aligned}
$$

Construct

$$
\begin{aligned}
\phi^{a \beta} & =\sum_{a b} \Gamma_{a b}^{a}\left(\Gamma_{a b}^{\beta}\right)^{*} \\
& =\sum_{\substack{a b \\
a^{\prime} b^{\prime}, a^{*} b^{\prime \prime}}} \mathscr{D}_{a a^{\prime}}^{+}(g) \Gamma_{a^{\prime} b^{\prime}}^{\alpha} \mathscr{D}_{b^{\prime} b}(g)\left(\mathscr{D}_{a a^{*}}^{+}(g) \Gamma_{a^{*} b^{*}}^{\beta} \mathscr{D}_{b^{*} b}(g)\right)^{*}
\end{aligned}
$$

tMarshak and Sudarshan (1961) and Macfarlane et al (1964) 
since

$$
\mathscr{D}^{+}(g) \mathscr{D}(g)=\mathscr{D}(g) \mathscr{D}^{+}(g)=1 .
$$

Now the invariance of $\mathscr{L}_{I}$ under $G$ demands

$$
\mathscr{D}_{a a^{\prime}}^{+}(g) \Gamma_{a^{\prime} b^{\prime}}^{a} \mathscr{D}_{b^{\prime} b}(g)=d_{a a^{\prime}}^{*}(g) \Gamma_{a b^{\prime}}^{a^{\prime}}
$$

Using this in (7) and making use of the unitarity of $d_{\alpha a^{\prime}}(g)$ we find,

$$
\phi^{\alpha \beta} d_{\beta \gamma}^{*}(g)=d_{\alpha \beta}^{*}(g) \phi^{\beta \gamma}
$$

Since $d(g)$ and therefore $d^{*}(g)$ is an irreducible representation this implies, by virtue of Schur's lemma $\phi^{\alpha \beta}$ must be a multiple of the identity matrix

$$
\phi^{\alpha \beta}=\sum_{a} \Gamma_{a b}^{a}\left(\Gamma_{a b}^{\beta}\right)^{*}=\phi \delta^{\alpha \beta}
$$

This concludes the proof.

Comment 1: This result holds good with all the $V_{\alpha}$ normalised uniformly so that $d_{a a^{\prime}}(g)$ is unitary, even if $\psi$ is not an irreducible representation as can be easily proved by inspection. The sum over $a, b$ now runs over all irreducible representations. Notice however that it is essential that the vector fields $V a$ belong to an irreducible representation as otherwise Schur's lemma will not apply to equation (10).

Comment 2: The above result can also be carried over when the interaction Lagrangian is invariant under algebraic structures other than a Lie group which are unitarily represented. With suitable modifications, it applies when the interaction is invariant under a graded Lie group rather than a Lie group. We note that Schur's lemma itself needs modification (see for example Rittenberg (1978) as follows. 'Let $R$ be an irreducible representation of a graded Lie algebra $S$, acting in a $Z_{2}$-graded vector space $V_{\overline{0}}+V_{\overline{1}}$ and $K$ a matrix which 'commutes' with all the generators of $S$, then either $K$ is a multiple of the unit matrix or if $\operatorname{dim} V_{\overline{0}}=\operatorname{dim} V_{\overline{1}}^{\prime} K$ can be a non-singular matrix which permutes $V_{\overline{0}}$ and $V_{\overline{1}}^{\prime}$. Nevertheless using a supertrace functional we can get an equivalent result (see Appendix).

Let us now return to the Lagrangian (3) and consider the effect of symmetry breakdown. As a consequence of the latter we are interested in knowing the coupling strength of the gauge fields which are eigenstates of the mass-matrix. Consider two such fields.

$$
\begin{aligned}
& A=\sum_{a} q^{a} V_{a} ; \sum_{a}\left|q^{a}\right|^{2}=1, \\
& B=\sum_{a} r^{a} V_{a} ; \sum_{a}\left|r^{a}\right|^{2}=1
\end{aligned}
$$


with $\quad \sum q^{a} r^{a}=0$.

which couple respectively to the following linear combination of the generators

$$
\begin{aligned}
& Q=\sum C_{\alpha} \Gamma^{\alpha} \\
& R=\sum D_{\alpha} \Gamma^{\alpha} .
\end{aligned}
$$

Clearly

$$
q^{\alpha}=C . C^{a}, \quad r^{a}=D . D^{a}
$$

where the normalisation constants $C$ and $D$ are given by

$$
C^{-2}=\sum_{a} C_{a}^{2}, \quad D^{-2}=\sum D_{a}^{2}
$$

Now the interaction Lagrangian (3) implies that the fields $A$ and $B$ have the couplings

$$
\mathscr{L}_{I}(A, B)=\sum_{\alpha} g \bar{\psi} \Gamma^{\alpha} q^{\alpha} \psi A+\sum_{\alpha} g \bar{\psi} \Gamma^{\alpha} r^{\alpha} \psi B
$$

where we have omitted the coupling of all the other gauge fields. Using Equations (12) to (17) in (18) we find

$$
\mathscr{L}_{I}(A, B)=g_{A} \bar{\psi} Q \psi A+g_{B} \bar{\psi} R \psi B
$$

with

$$
g_{A}=g C \text { and } g_{B}=g D
$$

Now it follows from (11) and (14), (15) and (17).

$$
\operatorname{Tr} Q^{2} / \operatorname{Tr} R^{2}=D^{2} / C^{2}
$$

which leads to the needed result

$$
g_{A}^{2} \operatorname{Tr} Q^{2}=g_{B}^{2} \operatorname{Tr} R^{2}
$$

The generators of interest in the following are (1) $Q=$ is electric charge operator so that $g_{A}^{2} / 4 \pi$ is to be identified with the fine structure constant. (2) $R=T_{3 L}$ the generator of the third component of the left handed weak-isospin, (3) $R=T_{3 C}$ the generator of the third component of the colour-isospin so that $g_{B}$ is the $Q C D$ strong coupling constant. (4) $R=T_{3 R}$, the generator of the third component of the right handed weak isospin related to the coupling of the weak intermediate vector bosons. 
It is clear from (22) that since $\operatorname{Tr} Q^{2}, \operatorname{Tr} T_{3 L}^{2}$ and $\operatorname{Tr} T_{3 C}^{2}$ for a given Fermion multiple are completely determined by a specification of electric charge, and their transformation under $\mathrm{SU}_{L}(2)$ and $\mathrm{SU}_{C}(3)$, the coupling constant relations are given by (22) do not depend on further details of the unifying algebra and do not depend either on the symmetry breaking mechanism or on how many stages of symmetry breaking there are for the model under consideration.

We note in passing that in deriving our results we have made no use of the gauge concept and therefore they can be used in contexts wider than those discussed here.

\section{Models of unifying algebra}

\subsection{Same fermions but different unifying algebras}

As an illustration, we consider the following set of Fermions $e_{L}^{-}, v_{L}^{e}, e_{R}^{-}, \mu_{L}^{-}, v_{L}^{\mu}, \mu_{R}^{-}$ and their antiparticles. The subscripts here denote the chiralities of the fields in question. We shall follow the canonical assignment $\left(v_{L}^{e}, e_{L}^{-}\right),\left(v_{L}^{\mu}, \mu_{L}\right)$ as $\mathrm{SU}_{L}(2)$ doublets and the rest as singlets.

Model A1. In one of the early attempts to fix the magnitude of $\theta_{w}$ Weinberg (1972) considered the following left-right symmetric model $\mathrm{SU}_{L}(3) \otimes \mathrm{SU}_{R}(3)$ where the states $\mu^{+}, v, e^{-}$form an SU (3) triplet. The neutrino here is a four component field with the identification.

$$
v_{L} \equiv v_{L}^{e}, \quad v_{R} \equiv v_{R}^{\mu C},
$$

where the superscript $C$ denotes the charge conjugate field. Defining with the help of (22)

$$
\sin ^{2} \theta_{w}=e^{2} / g_{L}^{2}=\operatorname{Tr} T_{3 L}^{2} / \operatorname{Tr} Q^{2}
$$

we find using the fact that $T_{3 L}$ is diagonal with eigenvalues $\pm \frac{1}{2}$ for the doublets and 0 for the singlets the result

$$
\sin ^{2} \theta_{w} \equiv \frac{1}{1} \operatorname{model} \mathrm{Al}
$$

Model A2. Recently Neeman (1979) and independently Fairlie (1979) have proposed a gauge model based on the graded Lie group $\mathrm{SU}(2 / 1)$ which contains the minimal $\mathrm{SU}_{L}(2) \otimes \mathrm{U}(1)$ as a sub-group. In this model $\left(v_{L}^{e}, e_{L}^{-}, e_{R}^{-}\right)$belong to the triplet representation of $\mathrm{SU}(2 / 1)$ while $\left(v_{L}^{\mu}, \mu_{L}^{-}, \mu_{R}^{-}\right)$belong to another independent triplet representation and similarly for every additional generation of leptons. Besides fixing the Weinberg angle, the model also fixes the Higgs coupling; however we shall be concerned with the former feature only. Again one has the canonical assignment of $\left(v_{L}^{e}, e_{L}^{-}\right)$to a doublet and $e_{R}^{-}$to a singlet under $\mathrm{SU}_{L}(2)$. As pointed out earlier (see also the Appendix) equation (11) is in general not valid for graded Lie groups. 
However in defining three-dimensional representation of $S U(2 / 1)$ we have the freedom to choose the relative normalisation of the isospin and hypercharge generators (the even part of the algebra) to be

$$
\operatorname{Tr}\left(\Gamma^{a} \Gamma^{b}\right)=\text { const } \delta^{a b}(a, b=1,2,3,8) .
$$

(valid for the defining representation only).

Using (26) it follows that the Weinberg angle is

$$
\sin ^{2} \theta_{w}=1 / 4 \quad \text { (model A2) }
$$

again as in model $\mathrm{A} 1$.

Digression: The SU(2/1) model has been extended to quarks also by Neeman and Thierry-Mieg (1979). The $(u, d)$ quarks belong to the quartet representation of $\mathrm{SU}(2 / 1)$ with $\left(u_{L}, d_{L}\right)$ as doublets and $u_{R}, d_{R}$ as singlets under $\mathrm{SU}(2)$. It is worth noting that for the quartet representation $\left(2^{6}\right)$ is not valid.

For the graded Lie algebra the Smushkevich relations (7) and (11) are replaced by the supertrace relations (see Appendix)

$$
\begin{aligned}
& \phi^{a \beta}=\operatorname{Str}\left(\Gamma^{a} \Gamma^{\beta}\right) \\
& \phi^{a \beta}=\phi \cdot \phi_{a d j}^{a \beta}
\end{aligned}
$$

where $\phi$ is a constant characteristic of the representation chosen and $\phi_{a d j}^{a \beta}$ is equation (28) for the adjoint representation. (We cannot normalise the supertraces since they are not necessarily positive definite). Equation (29) guarantees that the electric charge operator

$$
Q=T_{3} L+\cot \theta_{w} T_{p}
$$

\begin{tabular}{|c|c|c|c|c|c|c|}
\hline $\begin{array}{c}\text { Model } \\
\text { (cf, text) }\end{array}$ & Particles & $\begin{array}{c}\text { Electric } \\
\text { charge }\end{array}$ & $\mathrm{SU}_{L}$ (2) & $\sin ^{2} \theta_{w}$ & Type $^{+}$ & $\begin{array}{l}\text { Unifying } \\
\text { group }\end{array}$ \\
\hline Al & $e \nu \mu$ & $-1,0,+1$ & Two doublets & $1 / 4$ & 2 & $\mathrm{SU}_{R}(3) \times \mathrm{SU}_{L}(3)$ \\
\hline A2 & $\nu_{L}, e_{L}^{-}, e_{R}^{-}$ & $0,-1,-1$ & One doublet & $1 / 4$ & 1 & $\mathrm{SU}(2) / 1$ \\
\hline B1 & $\begin{array}{l}e \nu E \\
u, d, X\end{array}$ & $\begin{array}{l}-1,0,+1 \\
+2 / 3,-1 / 3,-1 / 3\end{array}$ & $\begin{array}{l}2 \text { leptons doublets } \\
3 \text { quark doublets }\end{array}$ & $\begin{array}{l}\text { ts } 5 / 16 \\
1 / 4\end{array}$ & 4 & SU(24) \\
\hline B2 & $\begin{array}{l}e_{\nu} E \\
u, d, X\end{array}$ & $\begin{array}{l}-1,0,+1 \\
+2 / 3,-1 / 3,-1 / 3\end{array}$ & $\begin{array}{l}1 \text { lepton doublet } \\
3 \text { quark doublets }\end{array}$ & $1 / 4$ & 4 & SU(24) \\
\hline $\mathrm{C} 1$ & $\begin{array}{l}e_{\nu e}^{\mu \nu}{ }_{\mu} \\
u, d, s, c\end{array}$ & $\begin{array}{l}-1,0,-1,0 \\
2 / 3,-1 / 3 \\
-1 / 3,2 / 3\end{array}$ & $\begin{array}{l}2 \text { lepton doublets } \\
6 \text { quark doublets }\end{array}$ & $3 / 8$ & 4 & SO(10) \\
\hline $\mathrm{C} 2$ & $\begin{array}{l}e \nu_{e} \mu \nu_{\mu} \\
u, d, s, c\end{array}$ & $\begin{array}{l}-1,0,-1,0 \\
\text { integrally charged }\end{array}$ & $\begin{array}{l}2 \text { lepton doublets } \\
6 \text { quark doublets }\end{array}$ & $3 / 8$ & 4 & {$[\mathrm{SU}(4)]^{4}$} \\
\hline
\end{tabular}

where $T_{y}$, is the generator of the U(1) subgroup, has the same value of $\cot \theta_{w}$ in every representation.

Table 1.

+ As defined in $\S 4$. 


\subsection{Same fermions with different $S U_{L}$ (2) properties}

In this subsection we shall consider the model variations built with essentially 12 Fermi fields which we shall denote by $u, d, X$ (each of which comes in three colours) and leptons $v, e^{-}, E^{+}$. The quarks $(u, d)$ belong to a doublet while the additional quark $X$ carrying charge $-1 / 3$ belongs to the trivial representation. For the leptons we have the following two options.

Model B1. (Fritzsch and Minkowski 1975) $\dagger$ Here the components of the neutrino field are identified as in model Al.

$$
\begin{aligned}
& \nu_{L}^{e}=\left(1+\gamma_{5}\right) \nu, \\
& \nu_{R}^{E C}=\left(1-\gamma_{5}\right) \nu .
\end{aligned}
$$

Clearly the 12 right handed and 12 left handed states can be embedded in SU(24) or any suitable subgroup which is also simple. To calculate the Weinberg angle we note that there are five left handed doublets (three $u, d$ ) quark doublets, $\left(v_{e}, e^{-}\right)$ and $\left(\nu_{E}, E^{-}\right)$so that

$$
\sin ^{2} \theta_{w}=\operatorname{Tr} T_{3 L}^{2} / \operatorname{Tr} Q^{2}=5 / 16 \quad \text { Model B1. }
$$

Model B2. Consider on the other hand the following assignment (Bajaj and Rajasekaran 1980a). The quarks have the same transformation as in Bl i.e. $(u, d)_{L}$ form a doublet and $X_{L}$ a singlet but now the lepton $E^{-}$is strictly a singlet with no associated neutrino. The left handed antineutrino $\bar{\nu}_{L}^{e}$ can be assigned to the trivial representation. Such schemes having extra leptons and quarks have been considered in literature in various other contents e.g. Khare et al 1979; Pandit 1976; Gupta and Mani 1974; Schecter and Ueda 1973) and it is possible to arrange these models in a way such that the interaction of the usual quarks and leptons remains largely unaltered.

To calculate the Weinberg angle we note that we have four $\mathrm{SU}_{L}$ (2) doublets, three from the $(u, d)$ colour triplet and one from $\left(\nu, e^{-}\right)_{L}$ so that

$$
\sin ^{2} \theta_{w}=\operatorname{Tr} T_{3 L}^{2} / \operatorname{Tr} Q^{2}=\left(4 \times \frac{1}{2}\right) / 8=1 / 4 \quad \text { Model B2 }
$$

It is clear that model B2 can be embedded in SU(24) as model B1, however the $\mathrm{SU}_{L}(2) \otimes U(1)$ subgroups which are identified to be the observed physical subgroups are obviously different in models B1 and B2 and hence lead to different values of $\boldsymbol{\theta}_{w}$.

The above discussion also suggests simple ways of inventing models with different values of $\theta_{w}$. For example to increase the value of $\theta_{w}$ one should either decrease the denominator $\operatorname{Tr} Q^{2}$ or increase the numerator $\operatorname{Tr} T_{3 L}^{2}$ in equation (33). The former is achieved by not allowing any left handed charged states which transform as singlets, in other words by deleting the quark $X$ and lepton $E$ in the above model. This leads us to the $S U(16)$ model with $S O(10)$ or $S U(5)$ as subgroups. We note that

tThese authors of course identify the $X$ quark with the strange quarks and the lepton $E^{+}$with $\boldsymbol{\mu}^{+}$. 
SO(10) and SU(5) are currently the most popular ones. It is easily seen that in this model there are four $\mathrm{SU}_{L}$ (2) doublets but $\operatorname{Tr} Q^{2}=16 / 3$ so that we have

$$
\sin ^{2} \theta_{w}=3 / 8 \text { SO (10) model. }
$$

Of course since the $\mathrm{SO}(10)$ model contains the $\mathrm{SU}(5)$ subgroup this result is already available at the SU(5) level for the 5 and the 10 representations separately. The other alternative of increasing the numerator is achieved in the $E_{\theta}$ model (Gürsey 1978). The quarks and leptons are assigned to two generations of the 27-plet representation of $E_{6}$ as follows:

$$
\begin{aligned}
& E_{6} \supset \mathrm{SU}_{L}(3) \times \mathrm{SU}_{R}(3) \times \mathrm{SU}_{C}(3) \\
& \text { (27) }=\left(\begin{array}{rrr}
-\hat{L}_{R} & -\hat{e}_{R} & \hat{E}_{R} \\
-e_{L} & \nu_{L} & -\hat{N}_{R} \\
E_{L} & -N_{L} & L_{L}
\end{array}\right)+\left(\overrightarrow{\mathscr{P}_{L}}, \vec{n}_{L}(\theta), n_{L}^{\prime}\right)+\left(\begin{array}{c}
\overrightarrow{\mathscr{P}}_{R}^{\prime} \\
\vec{n}_{R}^{\prime} \\
\vec{\Lambda}_{R}^{\prime}
\end{array}\right) \\
& \left(27^{\prime}\right)=\left(\begin{array}{rrr}
-\hat{L}_{R}^{\prime} & -\hat{\mu}_{R} & \hat{M}_{R^{v_{R}^{\prime}}} \\
-\mu_{L} & v_{L}^{\prime} & -N_{R}^{\prime} \\
M_{L} & -N_{L}^{\prime} & L_{L}^{\prime}
\end{array}\right)+\left(\overrightarrow{\mathscr{P}}_{L}^{\prime}, \vec{\Lambda}_{L}(\theta), \Lambda_{L}^{\prime}\right)+\left(\begin{array}{c}
\hat{\mathscr{P}}_{R} \\
\hat{n}_{R} \\
\hat{\Lambda}_{R}
\end{array}\right) \\
& n_{L}(\theta)=n_{L} \cos \theta+\Lambda_{L} \sin \theta ; \Lambda_{L}(\theta)=\Lambda_{L} \cos \theta-n_{L} \sin \theta \\
& \theta \equiv \text { Cabibbo angle. }
\end{aligned}
$$

The subgroup $\mathrm{SU}_{L}(3)$ acts on horizontal vectors while $\mathrm{SU}_{R}(3)$ acts on vertical vectors. The notation is $\hat{\psi}_{R}=\left(\psi^{c}\right)_{L} ; \hat{\psi}_{L}=\left(\psi^{c}\right)_{R}$. The leptons $e, \mu, E, M$ carry charge-1 while $L, \nu, N, L^{\prime}, \nu^{\prime}, N^{\prime}$ are neutral. It is easy to see that the quark structure is the same in models $\mathrm{B} 1$ and $\mathrm{B} 2$. However the number of $\mathrm{SU}_{L}(2)$ doublets have been increased by introducing neutral four component leptons $L$ and $N$, with the result

$$
\sin ^{2} \theta_{w}=\frac{\operatorname{Tr} T_{3 L}^{2}}{\operatorname{Tr} Q^{2}}=\frac{12 \times \frac{1}{2}}{16}=\frac{3}{8} \quad E(6) \text { Model }
$$

Of course equation (35) follows also trivially from $E(6) \supset S O(10) \supset S U(5)$. We now turn to a comparison of fractionally and integrally-charged quark models.

\subsection{Broken versus unbroken colour symmetry}

Consider now the following models with four leptons $\left(v_{e}, e^{-}, \nu_{\mu}, \mu^{-}\right)$and four quarks $(u, d, s, c)$ where the variation lies in the charge assignments to the quarks. In both models

$$
\left(\begin{array}{l}
\nu_{e} \\
e^{-}
\end{array}\right)\left(\begin{array}{l}
\nu_{\mu} \\
\mu^{-}
\end{array}\right)\left(\begin{array}{l}
u \\
d
\end{array}\right)\left(\begin{array}{l}
c \\
s
\end{array}\right)
$$


form doublets under $\mathrm{SU}_{L}(2)$. In the fractionally charged quark model we have the charge assignments $(u, d, s, c)=(2 / 3,-1 / 3,-1 / 3,2 / 3)$ while in the integrally charged Pati-Salam model (cf. Pati 1979, for a recent review) the assignments are more complex. However the isospin assignments remain the same. For the fractionally charged case it is easily seen

$$
\sin ^{2} \theta_{w}=3 / 8(\text { Model Cl) }
$$

For the integrally charged model, the definition of the mixing angle is different from (33). Bajaj and Rajasekaran (1980a) show that in the integrally charged case

$$
\sin ^{2} \theta_{w}=\frac{\operatorname{Tr} T_{3 L}^{2}}{\operatorname{Tr} T_{3 L}^{2}+\operatorname{Tr} T_{Y}^{2}}=3 / 8(\text { model C2) }
$$

where $T_{Y}$ is now the hypercharge generator. Note that with fractionally charged quarks we can use the group $S U(5)$ and $S O(10)$ for unification, while the integrally charged quark model can be embedded in [SU(4)] ${ }^{4}$ with reflection symmetry demanding all SU(4) couplings to be equal. However from a mathematical point of view both can be embedded in an SU(16) structure, with the physical content of the SU(16) groups being quite different in the two cases.

\section{Discussion}

When confronting the models considered in the previous section with neutral current experiments two questions are to be considered: (1) At what energies is the unification value for $\sin ^{2} \theta_{w}$ valid? (ii) How many neutral current generators or how many $Z$ bosons are there in the theory? Accordingly we may divide the models into four types:

Type 1. In these there is only one $Z$ boson. Besides quarks and leptons are in different multiplets. Such models do not relate the strong coupling constant with $g_{L}$ or $e$. Clearly the $\mathrm{SU}(2 / 1)$ (Model A2) is of this type. One may expect renormalisation corrections to be negligible and experimental consequence for the neutral current is practically the same as the minimal model with $\sin ^{2} \theta_{w}=0.25$.

Type 2. In these models quarks and leptons again belong to different representations of the unifying group. These models have several $Z$ bosons. For example in the model $A 1$ there are three $Z$ bosons. The experimental consequences can be expected to be slightly different from the prediction at the symmetry limit both due to renormalisation effects as well as mixing between the $Z$ bosons. However by careful planning one may be able to arrange things such that many of the currently known neutral current data are in agreement with the model (Georgi and Weinberg 1978; Bajaj and Rajasekaran 1979, 1980c).

Type 3. The SU(5) model is an example of the type of models where quarks and leptons are put in the same multiplet, but there is only one $Z$ boson. The symmetry value $\sin ^{2} \theta_{w}=3 / 8$ is expected to be valid only at enormous energies $M=10^{14}$ to $10^{16} \mathrm{GeV}$. The renormalisation corrections bring down the value of $\sin ^{2} \theta_{w}$ close to 
the experimental value of equation (2). (For a discussion with references to earlier literature see Bajaj and Rajasekaran 1980b).

Type 4. The $E_{6}$, SO(10), [SU(4)] ${ }^{4}$, etc. are all models which not only put quarks and leptons in the same multiplet but also have a large number of $Z$-bosons. As in type 3 the symmetry value is expected to be valid only at enormous energies. However interestingly enough in the [SU(4)] ${ }^{4}$ model, the broken $\mathrm{SU}_{c}(3)$ colour group can be chosen in such a way that unification can be achieved at energies of the order of $10^{5} \mathrm{GeV}$ itself (Pati 1979). The problems raised by the existence of several $Z$ bosons can be handled as in type 2 models.

In summary, we have shown that, some of the consequences of the various unification schemes depend only on the $\mathrm{SU}_{L}$ (2) transformation property of the observed leptons and quarks. There exists several models yielding for example the same value of $\sin ^{2} \theta_{w}$. Critical experiments on the proton life-time, the various neutral current experiments to test the existence of more than one $Z$ boson would soon be able narrow down the available choices and to select if at all one of these as the model for grand unification.

\section{Acknowledgements}

We are grateful to Prof. G Rajasekharan and Dr. J K Bajaj for communicating their results prior to publication and for several discussions without which this work could not have been carried out.

\section{Appendix-Lie superalgebras ${ }^{\dagger}$}

A superalgebra $A$ is a direct sum of two homogeneous algebras $A_{\bar{\delta}}$ and $A_{\bar{I}}$ with their degrees being respectively 0 and 1 and the algebraic operations preserve the degree modulo 2 . We write

$$
\begin{aligned}
& A=A_{\bar{\delta}}+A_{\overline{\mathrm{I}}} \\
& \operatorname{deg} a=\left\{\begin{array}{l}
0 \text { if } a \in A_{\bar{\delta}} \\
1 \text { if } a \in A_{\overline{\mathrm{I}}}
\end{array}\right.
\end{aligned}
$$

Any element of unique degree is said to be homogeneous. For two homogeneous elements $a, b$ we define the bracket

$$
[a, b]=a b-(-1)^{(\operatorname{deg} a) \cdot(\operatorname{deg} b)} b a
$$

and extend it to the whole algebra by linearity.

A Lie superalgebra $G=G_{\bar{\delta}}+G_{\bar{I}}$ is a superalgebra with a bracket operation [ , ] which satisfies the relation

$$
\begin{aligned}
& {[a, b]=-(-1)^{(\operatorname{deg} a) \cdot(\operatorname{deg} b)}[b, a]} \\
& {[a,[b, c]]=[[a, b], c]+(-1)^{(\operatorname{deg} a) \cdot(\operatorname{deg} b)}[b,[a, c]] .}
\end{aligned}
$$

tV G Kac (1977) contains a good discussion of the subject. 
Representations of $G$ are subsets of endomorphisms of a graded vector space $V=V_{\overline{0}}+V_{\bar{I}}$ onto itself with (i) the grading of $V$ being preserved under all homogeneous transformation of degree 0 , i.e.

$$
\begin{aligned}
& G_{\bar{\delta}} V_{\bar{\delta}} \subseteq V_{\bar{\delta}} \\
& G_{\bar{\delta}} V_{\overline{1}} \subseteq V_{\overline{1}}
\end{aligned}
$$

and (ii) the two subspaces $V_{\bar{\sigma}}$ and $V_{\bar{T}}$ are intertwined under homogeneous transformations of degree 1 , i.e.

$$
\begin{aligned}
& G_{\bar{I}} V_{\bar{\delta}} \subseteq V_{\bar{I}} \\
& G_{\bar{I}} V_{\bar{I}} \subseteq V_{\bar{\delta}}
\end{aligned}
$$

For a general element $a$ which has the matrix representation $a=\left(\begin{array}{ll}a & \beta \\ \gamma & \delta\end{array}\right)$ with $a$ a square matrix with the dimension of $V_{\overline{0}}, \delta$ a square matrix with the dimension of $V_{\bar{I}}$ and $\beta, \gamma$ in general rectangular we define the supertrace of $a$ by

$$
\operatorname{Str} a=\operatorname{tr} a-\operatorname{tr} \delta
$$

The supertrace is invariant under a change from one homogeneous base to another.

We note the following properties of the bilinear form

$$
\phi(a, b)=\operatorname{Str}(a b)
$$

(1) Consistency $\operatorname{str}(a b)=0$ if $a \in G_{\overline{0}}, b \in G_{\overline{1}}$ and vice versa.

(2) Super symmetry-str $(a b)=(-)^{(\operatorname{deg} a),(\operatorname{deg} b)} \operatorname{str}(b a)$.

This property is obvious if both $a b, \in G_{\overline{0}}$; if one is in $G_{\bar{\delta}}$ and the other in $G_{\bar{I}}$ both sides vanish. If both $a, b, \in G_{\mathrm{I}}$ with $a=\left(\begin{array}{ll}0 & a \\ \beta & 0\end{array}\right), \quad b=\left(\begin{array}{ll}0 & \gamma \\ \delta & 0\end{array}\right)$ then

$$
\begin{aligned}
& \text { Str }(a b)=\operatorname{tr}(a \delta-\beta \gamma), \text { while } \\
& \text { Str }(b a)=\operatorname{tr}(\beta \gamma-a \delta)
\end{aligned}
$$

Consequently $\operatorname{Str}([a, b])=0$ in all cases

(3) Invariance $\operatorname{Str}([a, b] c)=\operatorname{Str}(a[b, c])$

Proof. we have

$$
[b, a c]=[b, a] c+(-1)^{(\operatorname{deg} a) \cdot(\operatorname{deg} b)} a[b, c]
$$

Take the supertrace of this equation and use the result in (2) above i.e. $\operatorname{Str}([b, a c]=0$ and Eq. (A. 4).

Irreducibility of a representation: In case of a Lie super algebra $G$, irreducibility of the representation $V$ means that there are no nontrivial $\left(Z_{2}\right.$-graded!) submodules. 
In detail this means that there is no invariant proper subspace $W \subset V$ with $W=W_{\bar{D}}+W_{\bar{I}}$ which transforms under $G$ as in (A. 8) and (A. 9).

Schur's lemma: Let $V=V_{\overline{0}}+V_{\overline{1}}$ be $Z_{2}$-graded vector space. The algebra End $V$ has a natural $Z_{2}$ grading; more over is associative. Therefore defining a bracket operation (A. 4) converts it into a Lie superalgebra $1(V)$. Now let $M$ be an irreducible set of operators of $1(V)$ and define the centre $C(M)=$ The set of elements of $M$ such that $[a, m]=0 \forall a \in 1(V), m \in M$

Then either

(1) $C(M)=$ identity or (2) $\operatorname{dim} V_{\bar{\delta}}=\operatorname{dim} V_{\bar{I}}$ and $C(M)=(1, A)$ where $A$ is a non singular operator

in $V$ which permutes $V_{\bar{\delta}}$ and $V_{\bar{T}}$. For details see Kac (1977).

Thus Schur's lemma is modified as mentioned in the text.

In the case of invariance under a Lie superalgebra, the Smushkevich relations have to be modified. If $a, b, c$ are elements of a superalgebra $G=G_{\bar{\delta}}+G_{\Gamma}$ acting on a $\mathrm{Z}_{2}$-graded space $V=V_{\overline{0}}+V_{\mathrm{I}}$ the bilinear form

$$
\operatorname{Str}(a b)=\phi(a, b)
$$

is invariant in the sense

$$
\phi([a, b] c)=\phi(a[b, c])
$$

Hence in every representation of a Lie superalgebra the generators $\Gamma^{a}$ satisfy the relations that the two-index symbol,

$$
\phi^{a \beta}=\operatorname{Str}\left(\Gamma^{a} \Gamma^{\beta}\right)
$$

is invariant; and is therefore proportional to the Killing form

$$
g^{\alpha \beta}=\operatorname{Str}(\operatorname{ad} \alpha \operatorname{ad} \beta)
$$

where ad $a, \operatorname{ad} \beta$ refer to the matrices in the adjoint representation of the Lie superalgebra.

More generally if $\Gamma_{a n}^{a}$ are the coupling matrices for any three representations $A_{a}, B_{n}, C^{a}$ invariant under a superalgebra then the two-index symbols,

$$
\begin{aligned}
\phi^{\alpha \beta} & =\operatorname{Str}\left(\Gamma^{a} \Gamma^{\beta}\right), \\
\psi_{a b} & =\sum_{a, n} \Gamma_{a, n}^{\alpha} \Gamma_{n b}^{+a}(-)^{\operatorname{deg} a}, \\
\chi_{m n} & =\sum_{a, \alpha} \Gamma_{m a}^{+a} \Gamma_{a n}^{a}(-)^{\operatorname{deg} a},
\end{aligned}
$$

are all invariant; and hence proportional to their values for any such fixed choice of generators. In particular

$$
\phi^{a \beta}=\phi g^{a \beta}
$$




\section{References}

Bajaj J K and Rajasekaran G 1979 Pramana 12397

Bajaj J K and Rajasekaran G 1980a Madras Univ. Preprint MUTP-79/13 to be published in Pramāna

Bajaj J K and Rajasekaran G 1980b Pramana 14411

Bajaj J K and Rajasekaran G (1980e) Madras University Preprint

Dydak 1979 as quoted by J. Ellis CERN TH-2723

Fairlie D 1979 Phys. Lett. B82 97

Fritzsch H and Minkowski P 1975 Ann. Phys. (NY) 93193

Georgi H and Glashow S L 1974 Phys. Rev, Lett. 32438

Georgi H and Weinberg S 1978 Phys. Res. D11 1313

Glashow S L 1961 Nucl. Phys. 22579

Gupta V and Mani H S 1974 Phys. Rev. D10 1310

Gursey F 1978 Spring-Verlag lecture notes in Physics, Vol. 94, p 508

Kac V G 1977 Adv. Math. 268

Khare A, Mani H S and Ramachandran G 1979 Preprint, IP-BBSR/79-13

Macferlane A J, Mukunda N and Sudarshan E C G 1964 J. Math. Phys. 5576

Marshak R E and Sudarshan E C G 1961 Introduc tion to elementary particle physics (New York: Interscience)

Neeman Y 1979 Phys, Lett. B81 190

Neeman Y and Thierry-Mieg J 1979 Tel Aviv Preprint TAUP 727-79

Pandit L K 1976 Pramana 7291

Pati J C 1979 Technical Reprt. No. 80-003, Univ. of Maryland

Pati J C and Salam A 1973 Phys. Rev. D8 1240

Rittenberg V 1978 in Group theoretical methods in physics Proc. VI. Int. Conf. (Tubingen 1977). eds. O. Kramer and A Riekcers, Springer-Verlag Lectures Notes in Physics 79, Berlin-Heidelberg, New York

Salam A 1968 Elementary particle theory ed N Svartholm (Stockholm: Almquist and Wiksells) p. 367

Schecter J and Ueda Y 1973 Phys. Rev. D8 484

Weinberg S 1967 Phys. Res. Lett. 191264

Weinberg S 1972 Phys. Rev. D5 1962 